\title{
Investigation of 3D tungsten distributions in $(1,1)$ kink modes induced by toroidal plasma rotation
}

\author{
M. Weiland ${ }^{1}$, A. Gude ${ }^{1}$, V. Igochine ${ }^{1}$, M. Maraschek ${ }^{1}$, H. Zohm ${ }^{1}$, R. Bohle ${ }^{1}$, R. Dux ${ }^{1}$, K. \\ Lackner $^{1}$, T. Odstrčil ${ }^{1}$, T. Pütterich ${ }^{1}$ and the ASDEX Upgrade Team ${ }^{1}$ \\ ${ }^{1}$ Max-Planck-Institut für Plasmaphysik, 85748 Garching, Germany
}

Corresponding author: markus.weiland@ipp.mpg.de

\begin{abstract}
The presence of high-Z impurities, such as tungsten (W), can lead to non-uniform SXR radiation on flux surfaces due to centrifugal forces in rotating plasmas. The goal of the presented work is to characterize the effects of such rotation-induced radiation asymmetries on FFT-based SXR mode analysis. Therefore, a synthetic SXR diagnostic has been implemented, which takes into account the full 3D geometry of the detectors - resulting in a volume integration rather than the more simplifying line integration. We have focused on resistive $(1,1)$ kink modes, where we have implemented a model for the flux surfaces perturbed by the mode and the $\mathrm{W}$ distribution within. In a rotation scan, which leads to a variation of the asymmetry, a strong dependence of the FFT phase profile on the asymmetry strength is found. A comparison with experimental data shows good agreement, which verifies the used models.
\end{abstract}

\section{Introduction}

Controlling of magnetohydrodynamic (MHD) modes and understanding their behavior is an important premise for building a fusion reactor. A widely used method to investigate MHD modes is based on the measurement of Soft-X-Ray (SXR) radiation. It consists mainly of bremsstrahlung from electrons and ions, recombination radiation from low- $Z$ impurities, which both can be assumed to be constant on flux surfaces, and line radiation from heavy impurities (e.g. tungsten). In ASDEX Upgrade (AUG), it is observed by pinhole cameras with diodes and a beryllium filter and is commonly analyzed assuming a line integration measurement [1, 2]. To better understand the signals from SXR measurements, a synthetic SXR diagnostics has been developed at ASDEX Upgrade taking into account the full 3D geometry of the diagnostics. This results in the simulation of a volume of sight (VOS) for each detector diode. A comparison with the line of sight approach is performed and deviations between both are discussed.

The ASDEX Upgrade first wall is fully coated with tungsten (W), as this is a promising plasma facing component material for a future reactor. In terms of SXR data analysis, W plays a double role: At concentrations of $10^{-5}-10^{-4}$, tungsten can contribute a large part to the SXR radiation or even dominate it. In rotating plasmas, the $\mathrm{W}$ density is not 
constant on flux surfaces due to centrifugal forces, which can influence W transport [3] and leads to asymmetric SXR emissivities. In combination with the helical structure of MHD modes, fully three dimensional SXR emissivity distributions can occur. On the one hand, this makes the detection of MHD modes using SXR diagnostics more challenging. On the other hand, the SXR diagnostics also allows studying the tungsten impurity distribution and its correlation with MHD instabilities [4, 5.

To investigate the effect of tungsten on the SXR signals in the presence of MHD modes, a model to describe the 3D W-related SXR emissivities is developed and combined with the synthetic SXR diagnostic. The impurities, and hence the SXR emissivity described by this model, tend to accumulate on the low field side of each mode flux surface. A measure for the strength of this 3D effect is the rotation frequency. By varying it in the simulation, the effect of the asymmetry strength on the SXR signals is investigated. For the verification of the presented models, a comparison with experimental data in the presence of a stationary $(1,1)$ kink mode is performed.

The paper is structured as follows: In section 2, the sources of SXR radiation and their modeling are briefly discussed. In section 3, the virtual diagnostic using the VOS approach is presented and compared to the simplifying LOS approach. In section 4, we describe our model for resistive $(1,1)$ kink modes and in section 5 the model for the 3D W distribution inside this mode. The effect of the emissivity asymmetry on the SXR signals is characterized in section 6 and in section 7 the comparison with experimental data is presented.

\section{Modeling of soft $\mathrm{X}$-ray sources}

In fusion plasmas, soft X-rays in the range between 1 to $20 \mathrm{keV}$ emerge from bremsstrahlung as well as from recombination and line radiation. The spectrum $I(E)$ of bremsstrahlung between free electrons and ions is given by:

$$
I(E)=n_{\mathrm{e}}^{2} Z g_{\mathrm{ff}}\left(T_{\mathrm{e}}, E\right) \frac{\exp \left(-\frac{E}{T_{\mathrm{e}}}\right)}{\sqrt{T_{\mathrm{e}} / \mathrm{keV}}} \cdot 4.8566 \cdot 10^{-37} \mathrm{Wm}^{3} / \mathrm{keV}
$$

Here, $E$ is the energy of the emitted photon, $Z$ is the charge of the considered ion species and $g_{\mathrm{ff}}$ is the Gaunt factor, which describes deviations due to quantum mechanical effects. In the SXR range, $g_{\mathrm{ff}}$ lies close to 1 and can be computed according to [6]. The sum of recombination (free-bound) and line radiation (bound-bound) for any impurity element with charge $Z$ is proportional to its density $n_{\mathrm{Z}}$ and the electron density $n_{\mathrm{e}}$,

$$
I(E) \propto n_{\mathrm{e}} n_{\mathrm{Z}} f_{\mathrm{Z}}\left(E, T_{\mathrm{e}}\right)
$$

where $f_{\mathrm{Z}}\left(E, T_{\mathrm{e}}\right)$ contains the specific spectrum for that element and a given electron temperature $T_{\mathrm{e}}$.

The ratio between these creation channels depends largely on $Z$ and $T_{\mathrm{e}}$. Under typical tokamak conditions in the core plasma, light elements are fully stripped, and therefore hardly emit line and recombination radiation. Hence, bremsstrahlung dominates for low- 
Z elements and high temperatures. High-Z impurities, like $\mathrm{W}$, in contrast are not fully stripped, which is why they dominantly emit line radiation. The boundary between these cases is typically marked by oxygen $(\mathrm{Z}=8)$, for which the two creation channels match each other at $T_{\mathrm{e}} \approx 2.2 \mathrm{keV}$. The most common low-Z impurities at ASDEX Upgrade are boron (due to regular boronizations) and carbon, and thereby lie all below this boundary. Thus, their line and recombination radiation contribute only a small part to the total SXR radiation, which is favorable, since the densities of the different impurities are not routinely measured on AUG. To model it approximately, we calculate the impurity densities from $Z_{\text {eff }}$ measurements, assuming that only $\mathrm{B}$ and $\mathrm{C}$ impurities contribute to the value of $Z_{\text {eff }}$ (with equal concentrations).

For the high- $Z$ impurities, tungsten is the most important element in normal AUG discharge scenarios. Due to the fully W-coated first wall, its concentration can reach up to $10^{-4}$ and therefore contribute a significant part of the SXR radiation, or even dominate it [7, 8]. Still, the influence of tungsten on $Z_{\text {eff }}$ can be neglected at these concentrations.

The quantity, which can be observed by a SXR diagnostic at a position $\vec{x}$ is an integral of the spectral power density $I$ over the photon energy $E$, weighted with the spectral response function of the detector setup. We will call it emissivity $\epsilon$ in the following:

$$
\epsilon(\vec{x})=\int \operatorname{response}(E) \cdot I(E, \vec{x}) \mathrm{d} E
$$

The quantities that enter in the equations for low-Z elements are $n_{\mathrm{e}}, T_{\mathrm{e}}, Z_{\text {eff }}$, which can be considered to be constant on flux surfaces. However, for the tungsten density this is not necessarily true. In rotating plasmas, ions undergo a centrifugal force. Due to the high atomic mass of $\mathrm{W}$, this can lead to significant $\mathrm{W}$ density asymmetries on a flux surface. Therefore, we will model the SXR emissivity as a sum of the low-Z contribution $\epsilon_{0}\left(n_{\mathrm{e}}, T_{\mathrm{e}}, Z_{\text {eff }}\right)$, which is assumed to be a flux function, and the $\mathrm{W}$ contribution $\epsilon_{W}\left(n_{\mathrm{e}}, n_{\mathrm{W}}(R), T_{\mathrm{e}}\right)$, which can be asymmetric on flux surfaces (written as dependence on the major radius $R$ ). The former is fully determined by the experimentally measured profiles, while for the latter, the flux-surface averaged tungsten density profile $\left\langle n_{\mathrm{W}}\right\rangle$ remains as free parameter. The shape of the $\mathrm{W}$ distribution on a flux surface is modeled as described in section 5 .

\section{The SXR diagnostic at ASDEX Upgrade and its synthetic counterpart}

The Soft-X-Ray diagnostic at ASDEX Upgrade has a total number of 208 lines of sight, arranged at seven different poloidal camera positions (see fig. 11) [9]. Each camera has the same setup, consisting of a pinhole and a planar, linear array of Si-diodes. Photons with energies roughly below $1 \mathrm{keV}$ are filtered by a $75 \mu \mathrm{m}$ beryllium foil.

The pinhole-diode geometry results in a volume of sight (VOS) for each diode. This volume is relatively narrow in the poloidal direction, but largely spread in the toroidal direction. In order to take these effects into account, a synthetic diagnostic has been developed including the full 3D geometry of the VOS. To this end, an integration grid 


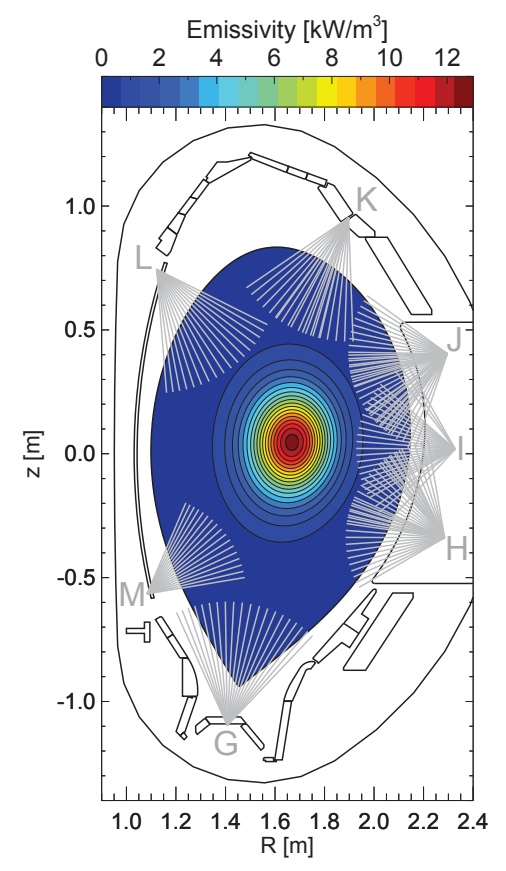

Figure 1: Poloidal cross-section of ASDEX Upgrade with all SXR lines of sight. The emissivity profile, which was used for the comparison between VOS and LOS calculations, is shown as contour plot.

with lattice planes parallel to the diode surface is used. The grid points inside one lattice plane are aligned in accordance to the mid point rule. The signal of one diode is then given by the volume integration:

$$
S_{\text {diode }}=\int_{\vec{x} \in \operatorname{VOS}} \epsilon(\vec{x}) \frac{\Omega(\vec{x})}{4 \pi} \mathrm{d} V \quad \text { Unit: [W] }
$$

$\Omega(\vec{x})$ is the solid angle with which the point $\vec{x}$ sees the diode. It is spanned by the four vectors in fig. 2 and can be calculated as described in [10].

The VOS approach is compared to the simplifying line of sight approach, assuming a typical, monotonically peaked SXR emissivity profile in the plasma (see fig. 3). For a horizontal view, only small differences are found: the more realistic VOS calculation gives 2-3\% higher signal than the LOS approach. However, for vertical views, large differences in both directions occur, ranging from $-3 \%$ to $+10 \%$.

By changing the pinhole dimensions in the synthetic diagnostics, it could be shown, that this deviation originates almost solely from the toroidal spreading of the VOS, whereas the poloidal spreading has a negligible effect. The resulting differences can be understood from fig. 4(a): Here, a top down view of the modeled emissivity in the mid-plane together with a cut of three example VOS is shown. In the VOS simulation, the signal of K_050, which looks through the HFS of the plasma, is about $7 \%$ higher as for the LOS integration. This is due to the toroidal curvature of the plasma, while the VOS has a rectangular shape. Therefore, K_050 sees higher emissivity in the edges of its VOS (originating from a more 


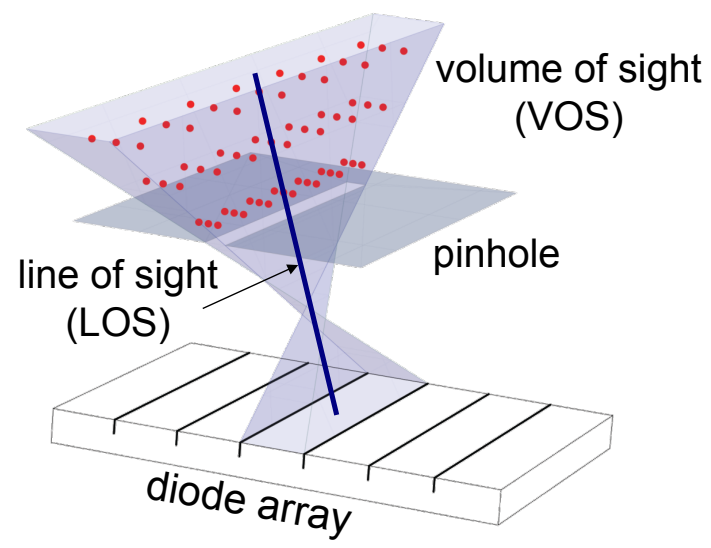

(a)

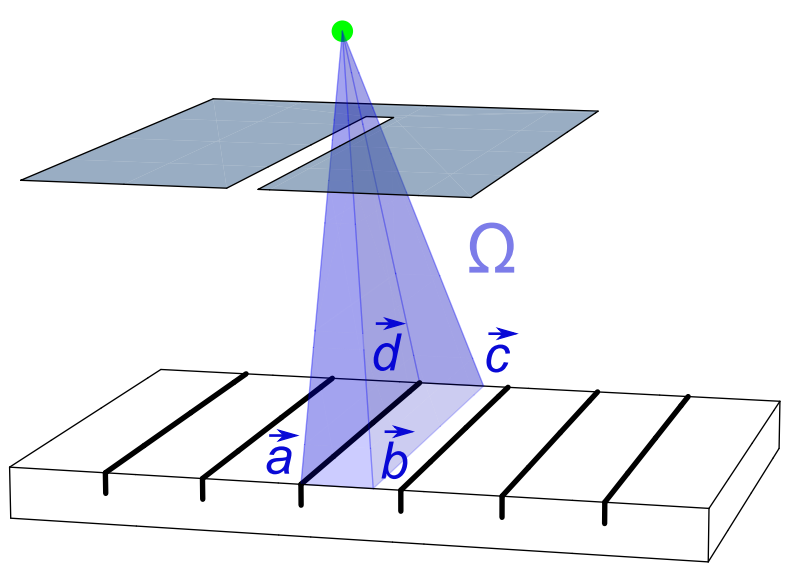

(b)

Figure 2: (a) Geometrical layout of the integration grid for the volume integration. (b) For each grid point, the solid angle $\Omega$ has to be calculated, with which the point views the detector diode. The diode can be partially shadowed by the pinhole. $\Omega$ is spanned by the four vectors $\vec{a}, \vec{b}, \vec{c}$ and $\vec{d}$, which form a skewed pyramid. [11]

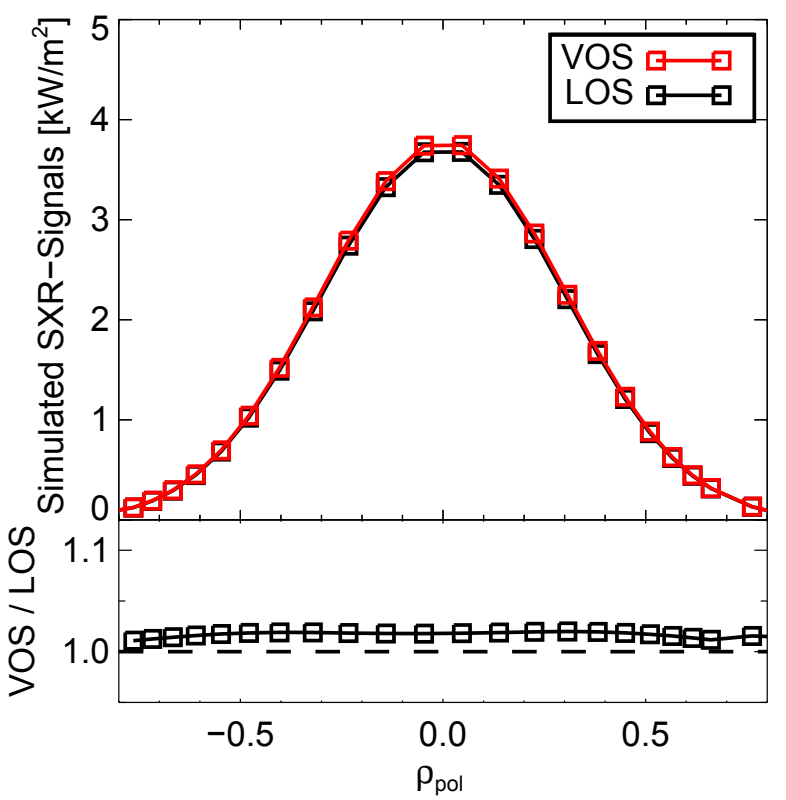

(a) Horizontal view (camera I)

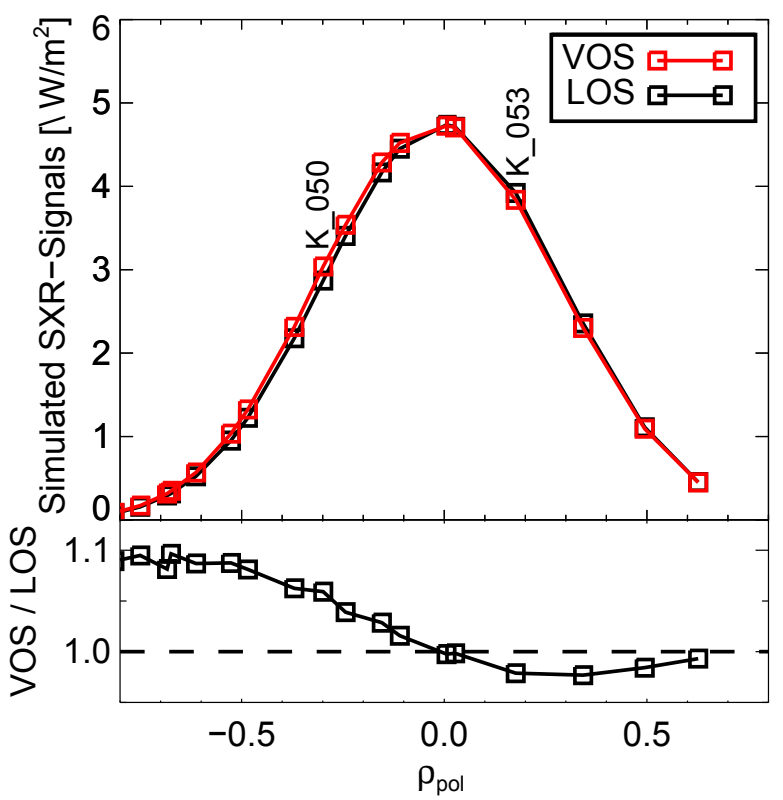

(b) Vertical view (camera $\mathrm{K}$ )

Figure 3: Comparison between simulated signals using the VOS and the approximate LOS approach. To see the difference more clearly, the ratio between the VOS and LOS signals is plotted in the lower part of the figures. The $x$-axis refers to the square root of the normalized poloidal flux $\rho_{\text {pol }}$ tangential to the LOS, where LOSs which have their tangential point in the upper part or the high field side of the plasma are assigned with negative values. 
central part of the plasma) than in the central part of the VOS, where one assumes its line of sight. Hence, one underestimates the signal when using the simplified LOS approach.

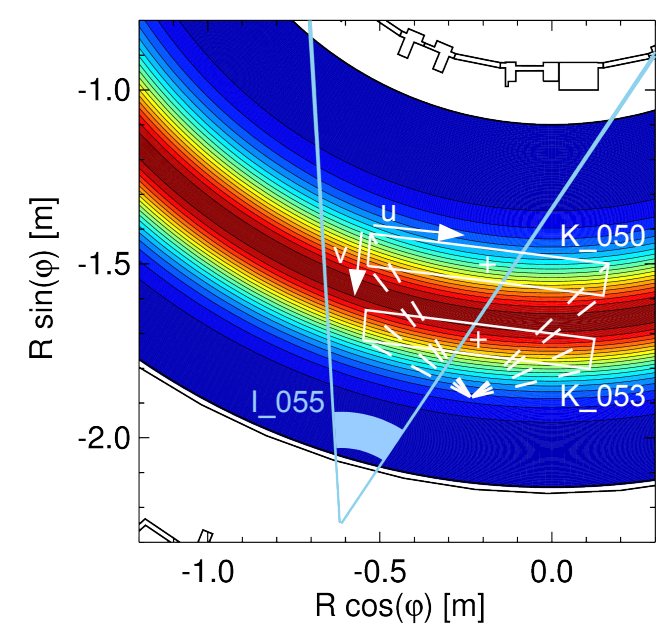

(a)

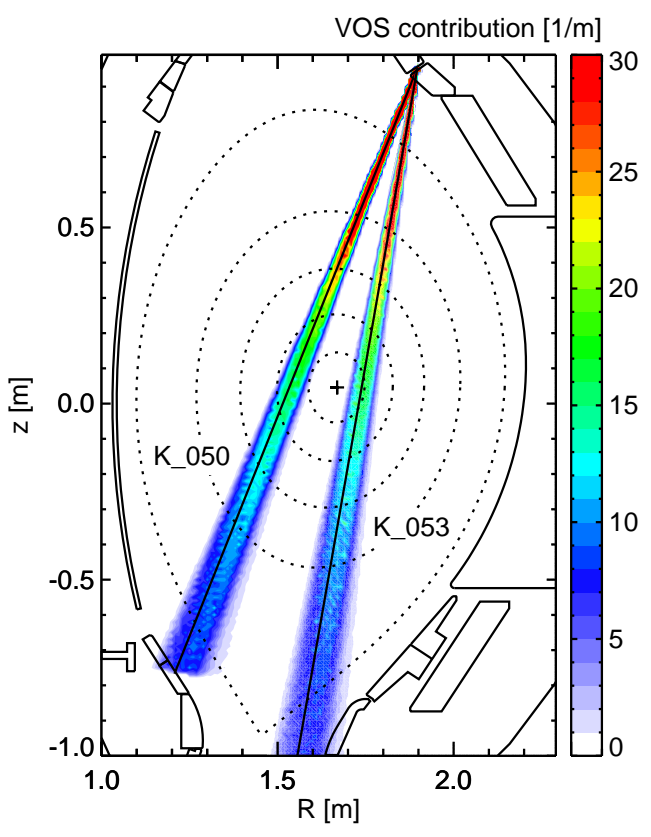

(b)

Figure 4: (a) Top down view at the mid-plane. The full volumes of sight of one horizontal (blue) and two vertical signals (white) are shown. For the latter, the corresponding lines of sight are marked with a cross. (b) Poloidal projection of the VOS contribution function $\operatorname{cont}(R, z)$ of $\mathrm{K}_{-} 050$ and $\mathrm{K}_{-} 053$ in comparison to the corresponding LOS. It is defined in such a way, that the signal of a $\operatorname{VOS}\left(\right.$ in $\left.\mathrm{W} / \mathrm{m}^{2}\right)$ is equal to $\int \operatorname{cont}(\mathrm{R}, \mathrm{z}) \epsilon(R, z) \mathrm{d} R \mathrm{~d} z$, and it has been calculated by making a $R-z$ histogram of the 3D integration grid. For both cases, the projection of the VOS is slightly shifted to the right (relative to the LOS).

For K_053, the situation is exactly opposite: Since it looks at the low field side, the toroidal curvature of the plasma acts in the different direction, leading to lower emissivity in the edges of the VOS than in its center. This is the reason why the LOS approach overestimates this signal by $3 \%$.

An alternative view on this feature is shown in figure 4(b), which shows the poloidal projection of the two VOS in comparison to their LOS. It can be seen, that the LOS does not lie centrally in the volume, but the volume is shifted towards the LFS. The shift is a consequence of projecting the toroidal spreading of the VOS into the poloidal plane. Together with the monotonically increasing emissivity profile which we have assumed, this results directly in a VOS/LOS ratio $>1$ on the HFS, and $<1$ on the LFS - in line with our previous findings. This radial shift is also important for tomographic reconstruction, because neglecting it leads to a systematic shift of the reconstruction and consequently to systematic errors if, for example, the magnetic axis should be determined from the tomography. This can be taken into account, by using a radially shifted LOS $R_{\mathrm{c}}(z)$ instead of the geometrical LOS $R_{\text {los }}(z)$. A good analytical approximation of $R_{\mathrm{c}}$ is presented in the appendix A. 
It has to be noted, that even stronger deviations could be expected for the $\mathrm{G}$ camera at the bottom of the machine, since it has an even more vertical orientation and a longer distance from the camera to the plasma center. However, on AUG, this camera looks through narrow slits in the divertor, which decrease the toroidal spreading of the VOS, compared to the $\mathrm{K}$ camera. If the $\mathrm{G}$ camera would have the same design as the $\mathrm{K}$ and $\mathrm{I}$ camera, our calculations predict roughly two times higher deviations (ranging from $-5 \%$ to $+20 \%)$.

The simulation result for the horizontal view can be explained by the same argumentation as above: On the low field side of the VOS the LOS approach yields too high signals, and on the HFS it yields too low signals. However, because of this horizontal arrangement, these deviations can cancel each other to a certain point. This explains, why the amplitude of the difference is much lower than for the vertical cameras. In the end, the contribution of the high field side is stronger, because there, the torus curvature is stronger and the VOS is spread more widely. Hence, one can explain, that the LOS simulation is overall lower than the VOS results for all signals of the horizontal view.

We can conclude for SXR diagnostics with similar design as the system on ASDEX Upgrade, that using the standard line of sight approach for SXR data analysis can lead to significant errors. Especially for vertical views, these errors can occur in both direction, ranging from $-5 \%$ to $20 \%$. This is for example important for tomography, where a precise relative calibration is needed. These toroidal effects can be taken into account by using a broad contribution function $\operatorname{cont}(R, z)$ as shown in fig. 4(b) instead of narrow lines of sight. A good approximation of $\operatorname{cont}(R, z)$ can be calculated, if the effect of the toroidal spreading is considered by shifting the LOSs according to equation 13 and by adding the poloidal spreading afterwards. Within this paper, we will use this approach for the tomography presented in section 7 and we will use the full VOS approach for all calculations of the synthetic diagnostic.

\section{Modeling of a resistive $(1,1)$ mode}

In resistive MHD, the finite resistivity of the plasma is considered, which allows the reconnection of magnetic field lines to islands. For the description of these resistive events, we use a model for the helical flux $\Psi^{*}$, which was published by Porcelli in [12] and which is based on the Kadomtsev sawtooth model [13]. In [12], a circular plasma cross-section is considered. To include a realistic, elongated plasma shaping, we apply this circular model in flux coordinates, with the square root of the normalized poloidal flux $\rho$ as radius and the straight field line angle $\theta^{*}[14,15]$ as angular coordinate in the poloidal plane. The mapping between flux and real space coordinates $\left(\rho, \theta^{*}\right) \leftrightarrow(R, z)$ is carried out based on CLISTE equilibrium reconstructions [16]. In the model, the plasma is divided into three areas (see fig. 5):

- Area I is the former center of the plasma. Due to the resistive $(1,1)$ kink mode, it is shifted with a constant displacement $\xi$. In flux coordinates, the flux surfaces are modeled as concentric circles, whose origin is shifted by $\xi$. The maximal radius of these circles is $\rho_{1}$. 


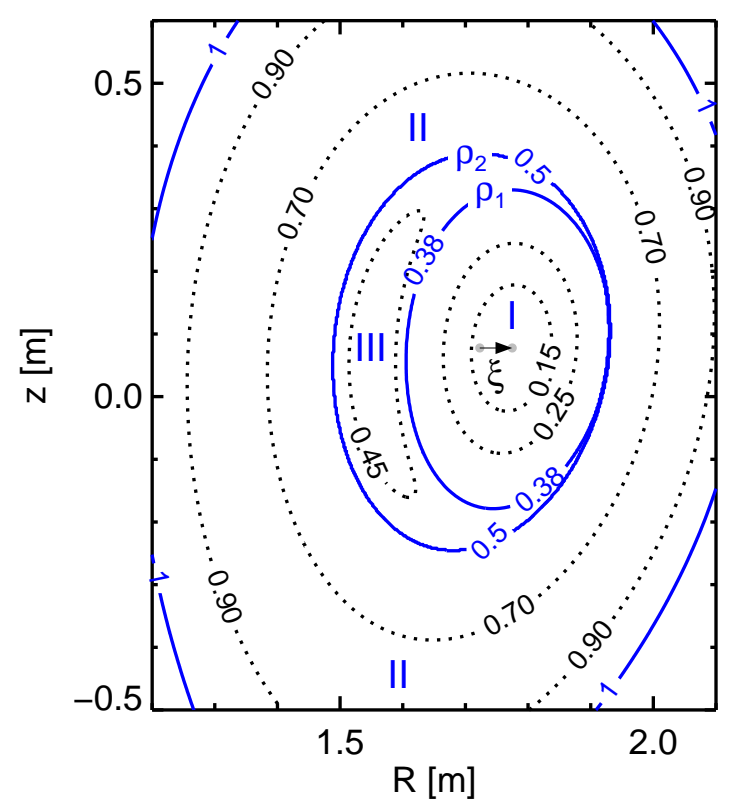

Figure 5: Modeled flux surfaces for a resistive $(1,1)$ kink mode, with the parameters $\rho_{2}=$ 0.5 and $\xi=0.12$. The contour lines correspond to the introduced coordinate $\rho_{\text {mod }}$, which describes the mode flux surfaces.

- Area II is the outer plasma, which is unperturbed by the mode. Hence, the flux surfaces form concentric circles (in flux coordinates) with a minimal radius $\rho_{2}$, with $\rho_{1}=\rho_{2}-\xi$.

- Area III defines the part, where magnetic reconnection has occurred and an island has formed. The resulting croissant shaped flux surfaces are described by the contour lines of a Hamiltonian $H$ (which means $\Psi^{*}=\Psi^{*}(H)$ ). $H$ is defined by:

$$
H=\frac{\left(\rho^{2}+\xi^{2}-2 \xi \rho \cos (\alpha)-\rho_{1}^{2}\right)\left(\rho_{2}^{2}-\rho^{2}\right)}{\rho^{2}+\xi^{2}-2 \rho \xi \cos (\alpha)}
$$

with: $\alpha=\theta^{*}-\varphi+\omega_{\mathrm{m}} t+$ const

Herein, $\theta^{*}$ is the straight field line angle, $\varphi$ the toroidal angle, and $\omega_{\mathrm{m}}$ the angular mode frequency. The contour of $H=0$ corresponds to the island separatrix. Inside the island $\mathrm{H}$ is positive and reaches its maximum at the $\mathrm{O}$-point of the island.

Within this model, we have two free parameters: $\xi$ and $\rho_{2}$. These need to be fitted to experimental data, while the island width is then given by $W=2 \xi$. It is convenient to describe all three areas with a unique flux label coordinate $\rho_{\text {mod }}$ from 0 to 1 , which we define as:

$$
\rho_{\text {mod }}:= \begin{cases}\sqrt{(\rho \cdot \cos \alpha-\xi)^{2}+(\rho \cdot \sin \alpha)^{2}} & \text { for area I }\left(\rho_{\text {mod }} \in\left[0 ; \rho_{1}\right]\right) \\ \rho_{1}+\frac{H}{\max (H)} \cdot\left(\rho_{2}-\rho_{1}\right) & \text { for area III }\left(\rho_{\text {mod }} \in\left[\rho_{1} ; \rho_{2}\right]\right) \\ \rho & \text { for area II }\left(\rho_{\text {mod }} \in\left[\rho_{2} ; 1\right]\right)\end{cases}
$$


The definition in area I resembles the shifted circles, and in area III, the Hamiltonian $H$ is scaled to match values between $\rho_{1}$ and $\rho_{2}$. It has to be noted, that $\rho_{\text {mod }}$ has a discontinuity due to the topology change caused by the magnetic island, as for $\epsilon \rightarrow 0$ with $\epsilon>0, \rho_{\text {mod }}=\rho_{2}-\epsilon$ describes the O-point of the island, while $\rho_{\text {mod }}=\rho_{2}+\epsilon$ is defined as the innermost flux surface of the unperturbed outer plasma.

\section{Modeling of the 3D tungsten distribution inside the mode structure}

In order to describe the W SXR emissivity $\epsilon_{\mathrm{W}}$, we need a model for the $\mathrm{W}$ density distribution $n_{\mathrm{W}}$ in the $(1,1)$ mode structure, since $\epsilon_{\mathrm{W}}$ on a mode flux surface is proportional to $n_{\mathrm{W}}$. The $\mathrm{W}$ density distribution can be asymmetric on (mode) flux surfaces due to centrifugal forces, which are induced by the toroidal plasma rotation (with an angular frequency $\left.\omega_{\text {rot }}\right)$. The distribution of high-Z trace impurities in an axisymmetric flux surface has been derived in [17, 18]. In [18], the force balance for the impurity ions parallel to the magnetic field is evaluated: The centrifugal force is balanced by the pressure gradient force and the electric field force, which arises because the much lighter electrons remain unaffected by the centrifugal force. In [17], a derivation based on neoclassical theory is performed. Both approaches yield the same result, where the $n_{\mathrm{W}}$ distribution on a flux surface with label $\rho$ is given by:

$$
\begin{array}{r}
n_{\mathrm{W}}(\rho, R)=n_{\mathrm{W}, \mathrm{ref}}(\rho) \cdot \mathrm{e}^{\lambda(\rho) \cdot\left(R^{2}-R_{\mathrm{ref}}^{2}\right)}=\left\langle n_{\mathrm{W}}\right\rangle(\rho) \cdot \frac{\mathrm{e}^{\lambda(\rho) \cdot R^{2}}}{\left\langle\mathrm{e}^{\left.\lambda(\rho) \cdot R^{2}\right\rangle}\right.} \\
\quad \text { with: } \lambda(\rho)=\left(1-\frac{T_{\mathrm{e}}}{T_{\mathrm{i}}+T_{\mathrm{e}}} Z_{\mathrm{W}} \frac{m_{\mathrm{i}}}{m_{\mathrm{W}}}\right) \frac{m_{\mathrm{W}} \omega_{\mathrm{rot}}^{2}}{2 T_{\mathrm{W}}}
\end{array}
$$

$T_{\mathrm{e}}, T_{\mathrm{i}}, T_{\mathrm{W}}$ are the electron, ion and tungsten temperatures, while $m_{\mathrm{i}}$ and $m_{\mathrm{W}}$ are the corresponding atomic masses. $Z_{\mathrm{W}}$ is the average charge of the tungsten ions, which we calculate from $T_{\mathrm{e}}$ assuming an ionization equilibrium, as described in [7]. There is no direct measurement of $T_{\mathrm{W}}$, so we will assume $T_{\mathrm{W}}=T_{\mathrm{i}}$, due to the high collisionality between $\mathrm{W}$ and the main ions. Since all these quantities entering in the prefactor $\lambda$ can be assumed to be constant on flux surfaces, $\lambda$ itself is a function of any flux coordinate. The asymmetry in the distribution originates from the major radius $R$, with $R_{\text {ref }}$ being an arbitrarily chosen reference point with the density $n_{\mathrm{W}, \mathrm{ref}}$. The latter is however not a good choice for an input parameter, because the total tungsten abundance on a given flux surface changes if $\lambda$ changes (e.g. in a rotation scan as in section 6), if $n_{\mathrm{W} \text {,ref }}$ is kept constant. Therefore, we have introduced a normalization with the flux surface average $<>$ in the second step. The brackets $<>$ are defined as:

$$
\langle x\rangle=\int_{0}^{2 \pi} \int_{0}^{2 \pi} x \cdot J d \theta^{*} d \varphi / \int_{0}^{2 \pi} \int_{0}^{2 \pi} J d \theta^{*} d \varphi
$$


with $J$ being the Jacobian of the $\left(\rho, \varphi, \theta^{*}\right)$ coordinate system. Hence, $\left\langle n_{W}\right\rangle$ is the flux surface averaged tungsten density, which can be used as an input parameter. By this formulation, the equation becomes independent of the reference point $R_{\text {ref }}$.

We now want to apply this equation to non-axisymmetric mode flux surfaces. Therefore, we have to assume, that the tungsten distribution reaches a quasi-stationary equilibrium inside the mode structure: First, the growth rate of the mode has to be small, compared to the time scale $\tau_{\mathrm{W} \text {,eq }}$ under which the tungsten distribution reaches its equilibrium inside a flux surface, parallel to the field lines. It can be assessed by the ratio of the half tokamak circumference and the average thermal velocity of W. For typical ASDEX Upgrade discharges $\left(T_{\mathrm{W}}=3 \mathrm{keV}, R_{0}=1.65 \mathrm{~m}\right)$ this gives $\tau_{\mathrm{W} \text {,eq }} \approx \pi R_{0} / \sqrt{\frac{8 k_{\mathrm{B}} T_{\mathrm{W}}}{\pi m_{\mathrm{W}}}}=82 \mu \mathrm{s}$, which is usually of the order of magnitude of the $(1,1)$ mode period, and well below the growth times. Second, the tungsten has to rotate with the same velocity as the mode structure, such that the relative rotation between both is zero. This is more critical, as the measured mode frequencies often differ by some $10 \%$ from the toroidal rotation frequency of light ions in the plasma center, obtained by charge exchange spectroscopy. However, it is not clear if this is also true for the tungsten rotation, which is not directly measurable by present-day diagnostics.

For the presented model, a compromise was made: Mode frequency and plasma rotation frequency are allowed to have (slightly) different values, such that the former can be set to the measured mode frequency, while the latter can be taken from the measured CXRS ion rotation profile. Effects from a possibly resulting rotation difference between the mode and tungsten are neglected.

We can describe the tungsten density distribution on mode flux surfaces then by applying equation 8 to the mode flux surfaces, which are defined by $\rho_{\text {mod }}=$ const. Consequently, all profiles (temperatues, $\left\langle n_{\mathrm{W}}\right\rangle$, rotation etc.) become functions of $\rho_{\text {mod }}$. The surface averaging refers then to an average on the three-dimensional mode flux surface. The Jacobian of our mode flux surface coordinate system cannot be described analytically anymore. Therefore, we have implemented a numerical calculation of the volume elements.

Figure 6 shows an example of the resulting $\mathrm{W}$ distribution on an island flux surface. It can be seen, that the tungsten avoids the part of the helical island flux surface, which is located on the high field side, while accumulating in the toroidal fraction of the flux surface, which is located on the low field side. Thus, this model results in both a poloidal and toroidal tungsten redistribution, leading to a fully three-dimensional distribution.

The SXR emissivity due to tungsten $\epsilon_{\mathrm{W}}$ is proportional to $n_{\mathrm{e}} \cdot n_{\mathrm{W}}$. Hence, we can describe it by exchanging $n_{W}$ by $\epsilon_{\mathrm{W}}$ in equation (8):

$$
\epsilon_{\mathrm{W}}(\rho, R)=\left\langle\epsilon_{\mathrm{W}}\right\rangle(\rho) \cdot \frac{\mathrm{e}^{\lambda(\rho) \cdot R^{2}}}{\left\langle\mathrm{e}^{\lambda(\rho) \cdot R^{2}}\right\rangle}
$$

Consequently, $\left\langle\epsilon_{\mathrm{W}}\right\rangle(\rho)$ is the mode surface averaged $\mathrm{W}$ emissivity profile, which we use as input parameter for our modeling. 


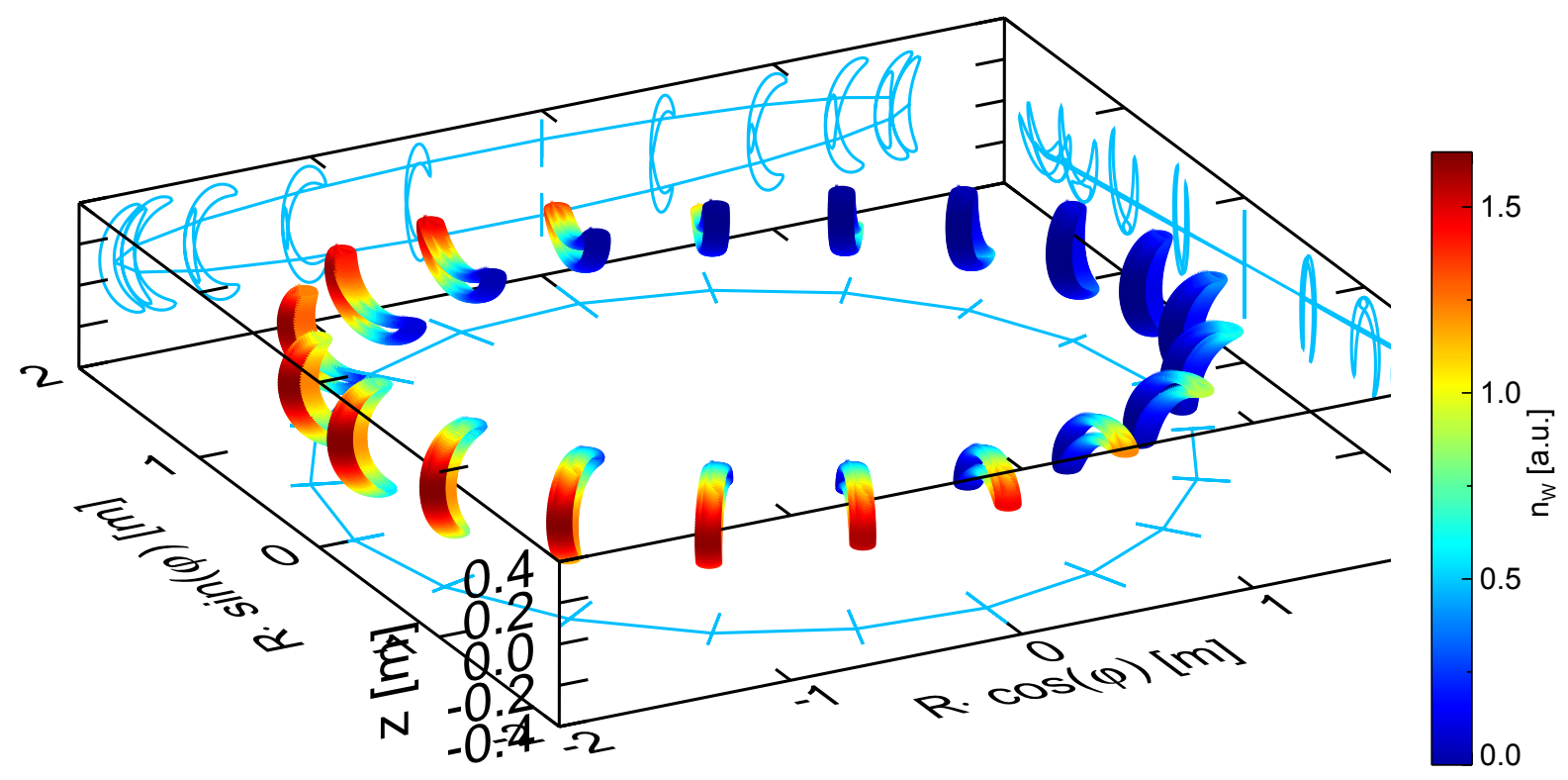

Figure 6: 3D-visualization of the modeled $\mathrm{W}$ density distribution on an island flux surface $\rho_{\text {mod }}=0.45$ at a toroidal rotation frequency of $f_{\text {rot }}=8 \mathrm{kHz}$. The parameters of the modeled kink mode are $\xi=0.12, \rho_{2}=0.5$. The surface averaged $\mathrm{W}$ density on the island flux surface is set to $\left\langle n_{\mathrm{W}}\right\rangle=1$. Without rotation $\left(f_{\text {rot }}=0\right)$, the $\mathrm{W}$ density would be constant on this island flux surface within our model.

\section{Characterization of SXR signals during a peaked $W$ emissivity profile}

In the following section, we want to characterize the influence of the rotation-induced 3D W distribution on SXR signals in the presence of a resistive $(1,1)$ mode. Therefore, an emissivity profile peaked in the plasma center was assumed, which is typical in ASDEX Upgrade discharges with the full-W wall. To see the pure effect, the emissivity was set to originate $100 \%$ from $\mathrm{W}$ for this case. The influence of the asymmetry was determined with a plasma rotation scan over four steps with $f_{\text {rot }}=[0 ; 6 ; 10 ; 12] \mathrm{kHz}$, which corresponds to tungsten Mach numbers $M_{\mathrm{W}}=2 \pi R_{0} f_{\text {rot }} / \sqrt{2 T_{\mathrm{i}} k_{\mathrm{B}} / m_{\mathrm{W}}}$ in the plasma center of $M_{\mathrm{W}}=$ $[0 ; 1.26 ; 2.10 ; 2.52] . f_{\text {rot }}=M_{\mathrm{W}}=0$ represents the case without any asymmetries. The input profiles are shown in figure 7. The resulting SXR emissivity distribution is shown in fig. 8 in the poloidal cross-section in three phases of the mode and for two $f_{\text {rot }}$ values. For $f_{\text {rot }}=12 \mathrm{kHz}$ the effects of the rotation are clearly visible. In phase $\Phi=180^{\circ}$, where the plasma center is shifted to the HFS, the total emissivity is lower than in the opposite phase $\Phi=0^{\circ}$, which is due to the toroidal redistribution in our model. Apart from that, the effect of the rotation is also visible in each phase alone, as the radiation maximum is not located on the shifted magnetic axis, but deviated towards larger $R$ values. For $f_{\text {rot }}=6 \mathrm{kHz}$ the same observations are made - yet much less distinct, since $\omega_{\text {rot }}$ enters quadratically in the exponential term in equation 8.

We want to study now the SXR signals resulting from this emissivity distribution, in particular the effects on the time averaged signal profiles, and the amplitude and phase 

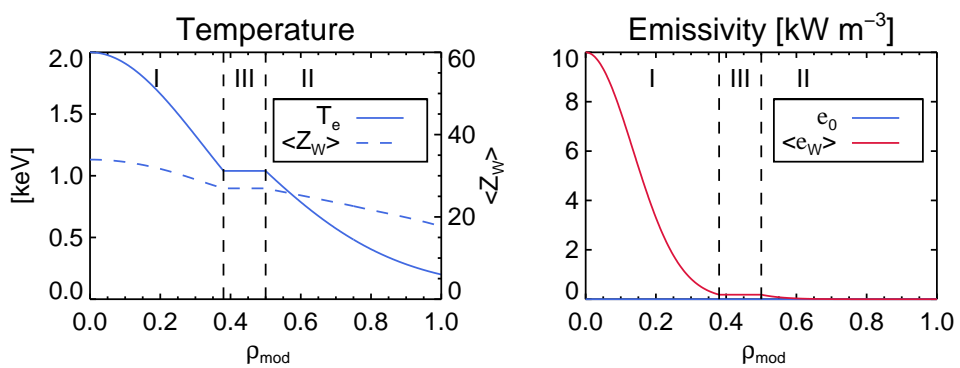

Figure 7: Input profiles for the modeling ( $T_{\mathrm{i}}$ is set equal to $T_{\mathrm{e}}$ ). The resistive kink is modeled with $\rho_{2}=0.5$ and $\xi=0.12$. The toroidal plasma rotation profile is assumed to be flat, and its value is varied within four steps $f_{\text {rot }}=[0 ; 6 ; 10 ; 12] \mathrm{kHz}$.
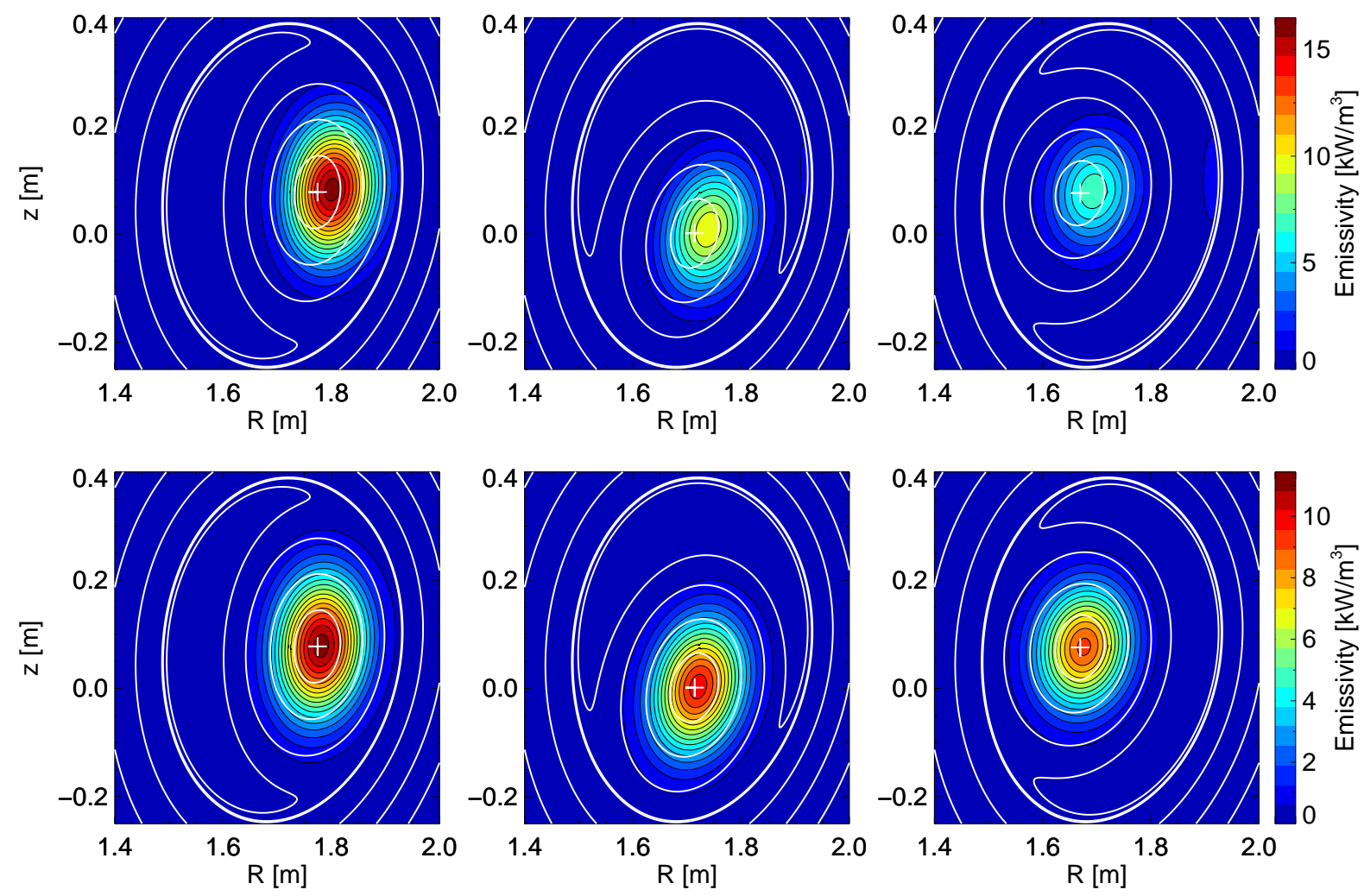

(a) $\Phi=0^{\circ}$

(b) $\Phi=90^{\circ}$

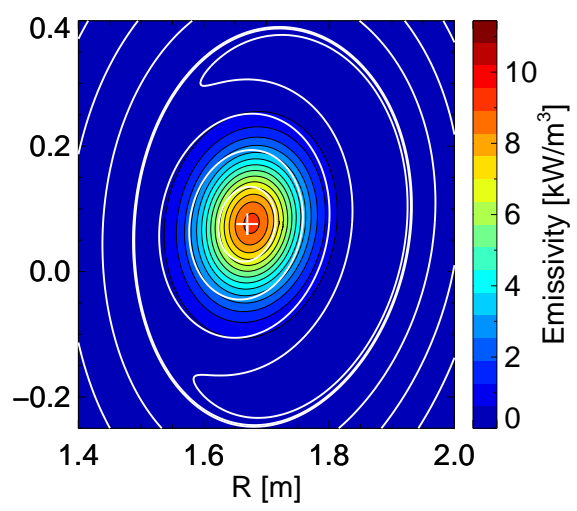

(c) $\Phi=180^{\circ}$

Figure 8: Contour plot of the modeled emissivity in the poloidal cross-section for two different toroidal rotation frequencies and in three different phases $\Phi$. Upper row: $f_{\text {rot }}=$ $12 \mathrm{kHz}$. Lower row: $f_{\text {rot }}=6 \mathrm{kHz}$. For comparison, the mode flux surfaces are plotted in white. In the case with no rotation, $f_{\text {rot }}=0 \mathrm{kHz}$, the emissivity contour lines are identical with the flux surfaces. 
profiles of a FFT analysis at the mode frequency. To see all effects clearly in the simulation, the spatial resolution of the diagnostic was increased by adding virtual LOS in between the real ones.
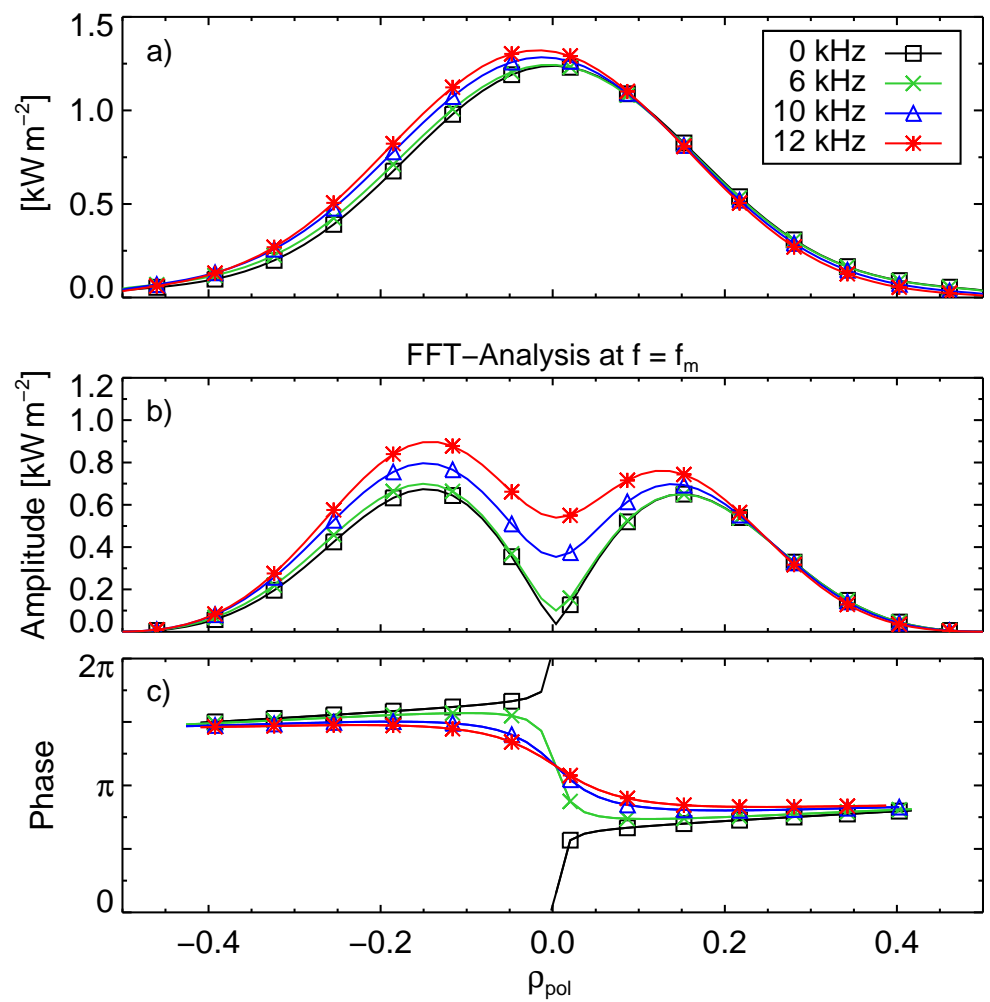

Figure 9: Forward modeled SXR signal profiles of the horizontal camera I for four toroidal rotation frequencies $f_{\text {rot }}=[0,6,10,12] \mathrm{kHz}$. a) Time-averaged signals. $\left.\mathrm{b}+\mathrm{c}\right)$ : Amplitude and phase profile from a FFT analysis of the simulated signals. The phase profile is plotted $2 \pi$ periodic. The symbols correspond to the actual LOS setup, while the full curve has been calculated by adding virtual LOS in between existing ones.

In figure 9 the calculated profiles are plotted for a horizontal SXR camera (I). The timeaveraged signals change hardly with the rotation frequency - which can be expected, since the rotation causes mainly a radial shift of the radiation, which cannot be resolved by a horizontal view. In contrast, the amplitude profile increases strongly in the plasma center. While for symmetric radiation (i.e. no rotation), the amplitude minimum is expected to be zero [19, 20], for strong rotation, the minimum is less pronounced and raised significantly. Since the horizontal view cannot resolve HFS-LFS asymmetries, this has to be caused by the toroidal radiation asymmetry. The rise of the central minimum is hence a measure for the strength of the toroidal tungsten redistribution. However, due to finite spatial resolution, the minimum cannot always be determined from experimental data.

An even clearer effect can be seen in the phase profile. This is in particular important, since the shape of the phase jump resulting from the $(1,1)$ mode has been proven to be very robust against any other parameter variation, such as changing the emissivity profiles 
or the mode displacement [11. With increasing rotation, the shape of the phase profile changes its direction around the phase jump. Hence, the shape of the phase profile is a clear indicator of the radiation asymmetry strength, which is also easier to detect with finite spatial resolution.

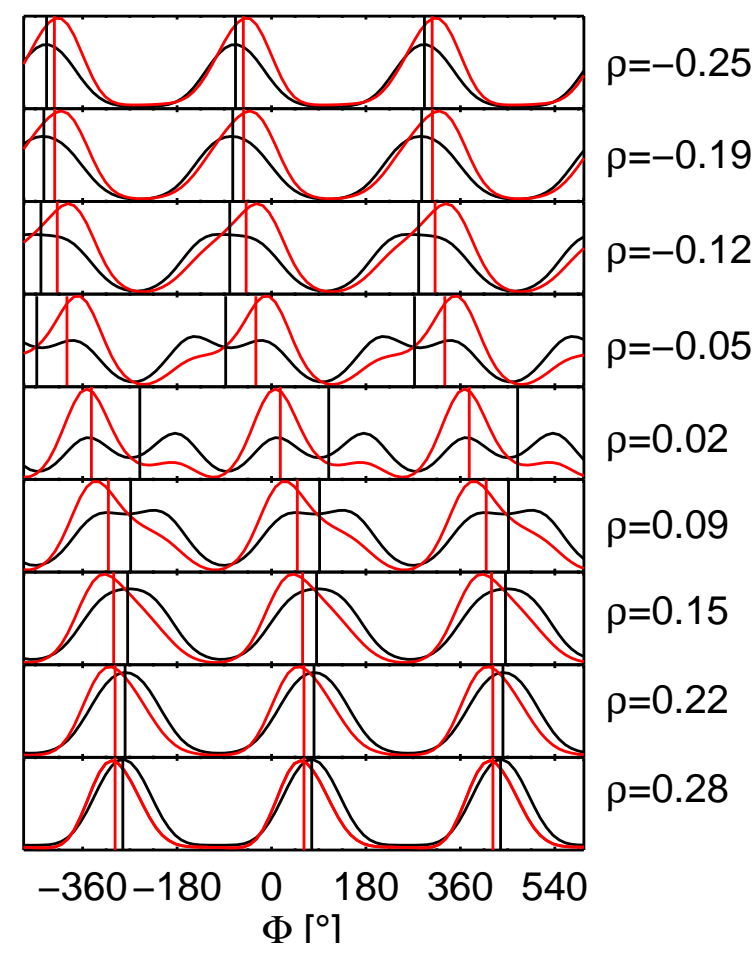

Figure 10: Time traces of the innermost signals of the horizontal camera I for the two extreme cases $\left(f_{\text {rot }}=0\right.$ (black) and $f_{\text {rot }}=12 \mathrm{kHz}($ red $\left.)\right)$. To allow comparison with fig. 8 , the $x$-axis is not labeled with the time, but with the mode phase $\Phi$. The phase from the FFT analysis of the time trace is plotted with vertical bars. It can be seen, that the time traces are not purely sinusoidal in general. This means, that the FFT-analysis also yields contributions from higher harmonics. For $f_{\text {rot }}=0$, the innermost signal $\rho=0.02$ oscillates even dominantly at the $2^{\text {nd }}$ harmonic of the mode frequency $\left(f=2 f_{\mathrm{m}}\right)$, while the FFT amplitude at the mode frequency is low (as seen in fig. 9 p.

The reason of this phase profile change can be explained by fig. 10, which shows the time traces of the innermost signals of the camera for the two extreme cases $\left(f_{\text {rot }}=0\right.$ and $f_{\text {rot }}=12 \mathrm{kHz}$ ). For comparison, the $\mathrm{x}$-axis is not labeled with the time, but with the mode phase (same as in fig. 8). It can be seen, that for the most central channel, the time trace corresponding to zero rotation has a double peak-shape. The peaks correspond to the phases, where the emissivity maximum (located at the kink-shifted plasma center) crosses the LOS. The peak at phase $180^{\circ}$ corresponds to the phase, where the plasma center is shifted towards the HFS. This peak is slightly higher, because the distances between neighboring flux surfaces are broader on the HFS than on the LFS due to the Shafranov-Shift. In the case with high rotation $\left(f_{\text {rot }}=12 \mathrm{kHz}\right)$, the situation is different: The peak at $\Phi=0^{\circ}$ is much larger, because the $\mathrm{W}$ emissivity is accumulated on the LFS. This difference influences the phase, which we get from the FFT for the fundamental 
frequency, and explains the phase profile changes with increasing rotation in the plasma center.
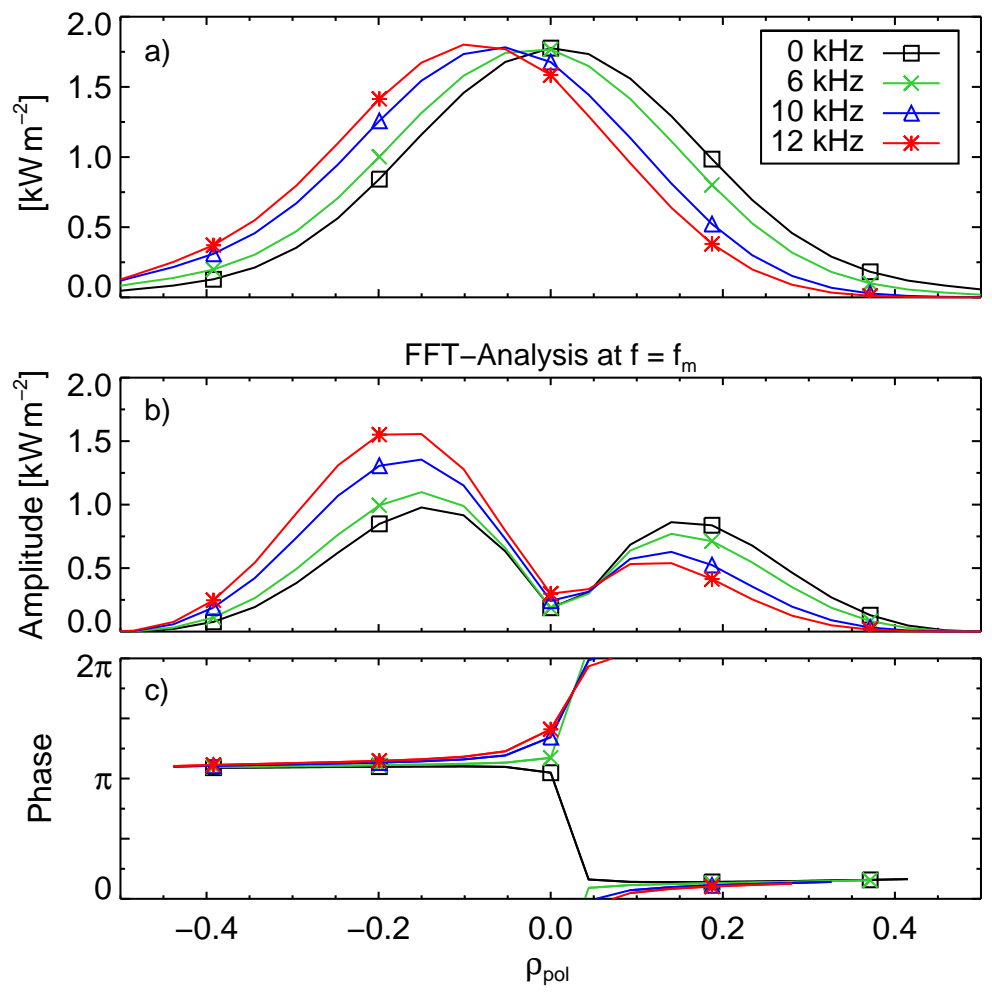

Figure 11: Forward modeled SXR signal profiles of camera $G$ for four toroidal rotation frequencies $f_{\text {rot }}=[0,6,10,12] \mathrm{kHz}$. a): Time-averaged signals. $\left.\mathrm{b}+\mathrm{c}\right)$ : Amplitude and phase profile from a FFT analysis of the simulated signals. The phase profile is plotted $2 \pi$ periodic.

Figure 11 shows the calculated profiles for a vertical view. Here, the poloidal redistribution dominates the signal profiles: With increasing rotation, the time averaged signal profile is shifted towards the LFS, and the amplitude profile is rising on the LFS and lowering on the HFS. In contrast to the horizontal view, the uplift of the central minimum is barely visible. The phase jump is also changing its direction, but the effect is less pronounced than for the horizontal view.

In this section, we have characterized the influence of rotation-induced SXR asymmetries on the FFT-profiles in the presence of a resistive $(1,1)$ kink mode. It can be argued, that these results are also valid for an ideal kink mode, since we have assumed a flat (i.e. feature-less) emissivity profile in the island, such that the island is not visible to the SXR diagnostic. 


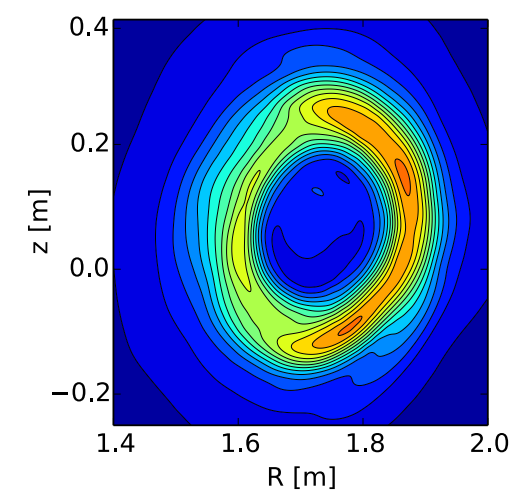

(a) $\Phi \approx 0^{\circ}$

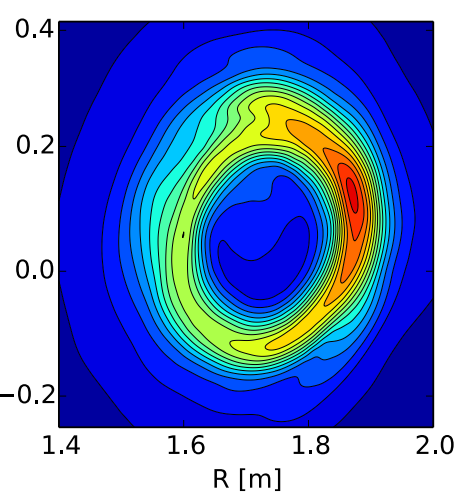

(b) $\Phi \approx 90^{\circ}$

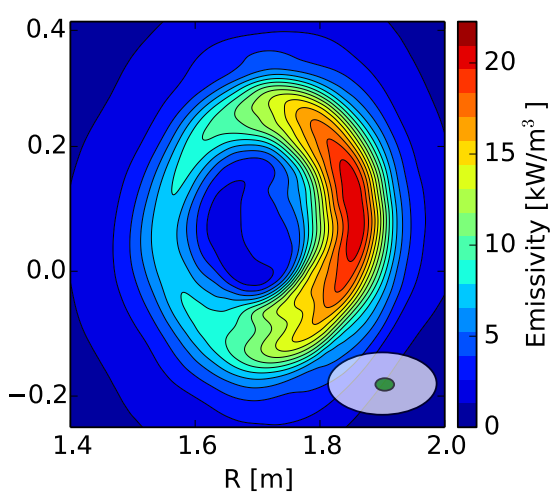

(c) $\Phi \approx 180^{\circ}$

Figure 12: Tomography of the experimental Soft-X-Ray data from discharge \#26299 at $t=3.648 \mathrm{~s}$ in three different phases $\Phi$ of the mode. The ellipses in the right corner of (c) show lower and upper bounds of the spatial resolution: The larger ellipse corresponds to the widths of the volumes of sight in the plasma center. The smaller ellipse corresponds to the reconstruction of a point-like emissivity dot.

\section{Comparison to experimental data in the presence of a hollow emissivity profile}

In the following section we want to check, if our model describes the tungsten distribution well, and if the signal features depicted in the last section are in line with experimental data. We have chosen the ASDEX Upgrade discharge \#26299 between 3.648 and 3.649s. The discharge was run with $B_{\mathrm{t}}=-2.474 \mathrm{~T}$ and a plasma current of $1 \mathrm{MA}$. The electron density in the core is $8.2 \cdot 10^{-19} \mathrm{~m}^{-3}$ and the plasma was heated with $7.5 \mathrm{MW} \mathrm{NBI}$ and 0.9 MW of ECRH. In the considered time interval, a saturated $(1,1)$ mode occurs between two sawtooth crashes. Figure 12 shows a tomographic reconstruction (based on [21]) of the SXR emissivity at three different phases of the mode. The center of the plasma, rotating because of the kink, can be identified as the blue spot in the middle. Thus, the emissivity profile is strongly hollow (i.e. it has a local minimum at the plasma center). Since the electron temperature and density are peaked, this hollow radiation profile can only be explained by a strong contribution from tungsten located around the resonant $q=1$ surface [22]. In phase (c) the reconstructed SXR radiation has its global maximum. Its croissant-like shape suggests, that we see here an $(1,1)$ island, whose O-point is located on the LFS with a strong tungsten accumulation around it. In the opposite phase (a), the X-point lies on the LFS. Indeed, we see there a local minimum of the radiation. A croissant-like shape around the O-point on the HFS is not visible - which can be explained by a toroidal redistribution of tungsten towards the more favorable phase, where the island O-point is on the LFS.

The strong asymmetry makes this a challenging test candidate for our model. Furthermore, we want to assess if the interpretation mentioned above matches the measured SXR signals quantitatively. Figure 13 shows the input profiles for our forward model. The kinetic profiles $\left(n_{\mathrm{e}}, T_{\mathrm{e}}, T_{\mathrm{i}}, v_{\text {rot }}, Z_{\text {eff }}\right)$ are taken directly from the experimentally obtained 

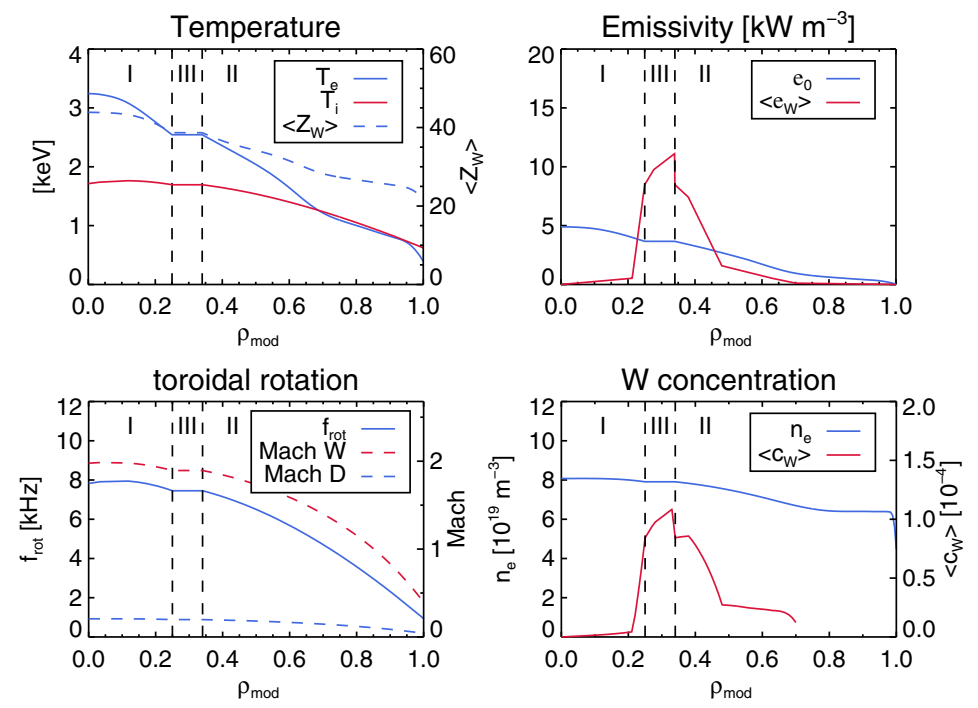

Figure 13: Input profiles for the forward-model. The parameters for the resistive kink are $\xi=0.09, \rho_{2}=0.34$. The mode frequency is $f_{\mathrm{m}}=10.23 \mathrm{kHz}$. The $Z_{\text {eff-profile is assumed }}$ to be constant with $Z_{\text {eff }}=1.86$, which is evaluated from spectroscopic bremsstrahlung measurements in the visible range. The profiles are plotted as a function of the mode coordinate $\rho_{\text {mod }}$ (see definition in section (4). Area III marks the inside of the island, with $\rho_{\text {mod }}=0.25$ being the island separatrix and $\rho_{\text {mod }}=0.34$ being the island O-point. The emissivity is continuous, since the right-sided limit (coming from area II) at $\rho_{\text {mod }}=0.34$ is equal to the value at $\rho_{\text {mod }}=0.25$. In addition to the rotation frequency, the Mach numbers $M_{\mathrm{i}, \mathrm{W}}=2 \pi R f_{\text {rot }} / \sqrt{2 k_{\mathrm{B}} T_{\mathrm{i}, \mathrm{W}} / m_{\mathrm{i}, \mathrm{W}}}$ for $\mathrm{D}$ and $\mathrm{W}$ are shown. The mode flux surface averaged $\mathrm{W}$ concentration $\left\langle c_{\mathrm{W}}\right\rangle=\left\langle n_{\mathrm{W}}\right\rangle / n_{\mathrm{e}}$ is calculated from the fit result $\left\langle\epsilon_{\mathrm{W}}\right\rangle$.

mode-averaged profiles. They were adapted for our modeling of the mode structure in such a way, that all profiles were set to be constant inside the island. The resulting low- $Z$ SXR emissivity $\epsilon_{0}$ is calculated based on these profiles, as described in section 2 and is constant on the mode flux surfaces. The remaining free parameters of our model are: the displacement of the $(1,1)$ mode, the radial extension of the mode $\rho_{2}$ and the mode flux averaged tungsten SXR emissivity $\left\langle\epsilon_{\mathrm{W}}\right\rangle$ profile. They were manually fitted to the experimental SXR signal profiles for the I camera, shown in figure 14. For $\left\langle\epsilon_{\mathrm{W}}\right\rangle$, a piecewise set of continuous, linear functions is chosen for the sake of simplicity. $\rho_{2}$ can be determined more or less directly from the width of the FFT amplitude profile $\left(\rho_{2}=0.34\right) .\left\langle\epsilon_{\mathrm{W}}\right\rangle$ and $\xi$ have then to be fitted to match the mode-averaged signal profile and the FFT-amplitude profile. Especially the amplitude profile is influenced by both, because the amplitude grows with increasing $\xi$, but also with increasing emissivity inside the mode. The shape of the phase profile is mostly unaffected by this fitting procedure, because the shape is determined by the strength of the asymmetry, which is given by the measured kinetic plasma profiles.

Figure 14 shows the result of the fit in comparison to the experimental data profile of the horizontal camera I, where we expect the strongest effect on the phase profile. Clearly, a good agreement between experiment and simulation can be observed. The shape of the phase jump is very well described by our model. For comparison, we have turned off the rotation in the simulation (plotted with a dashed line), which corresponds to a 

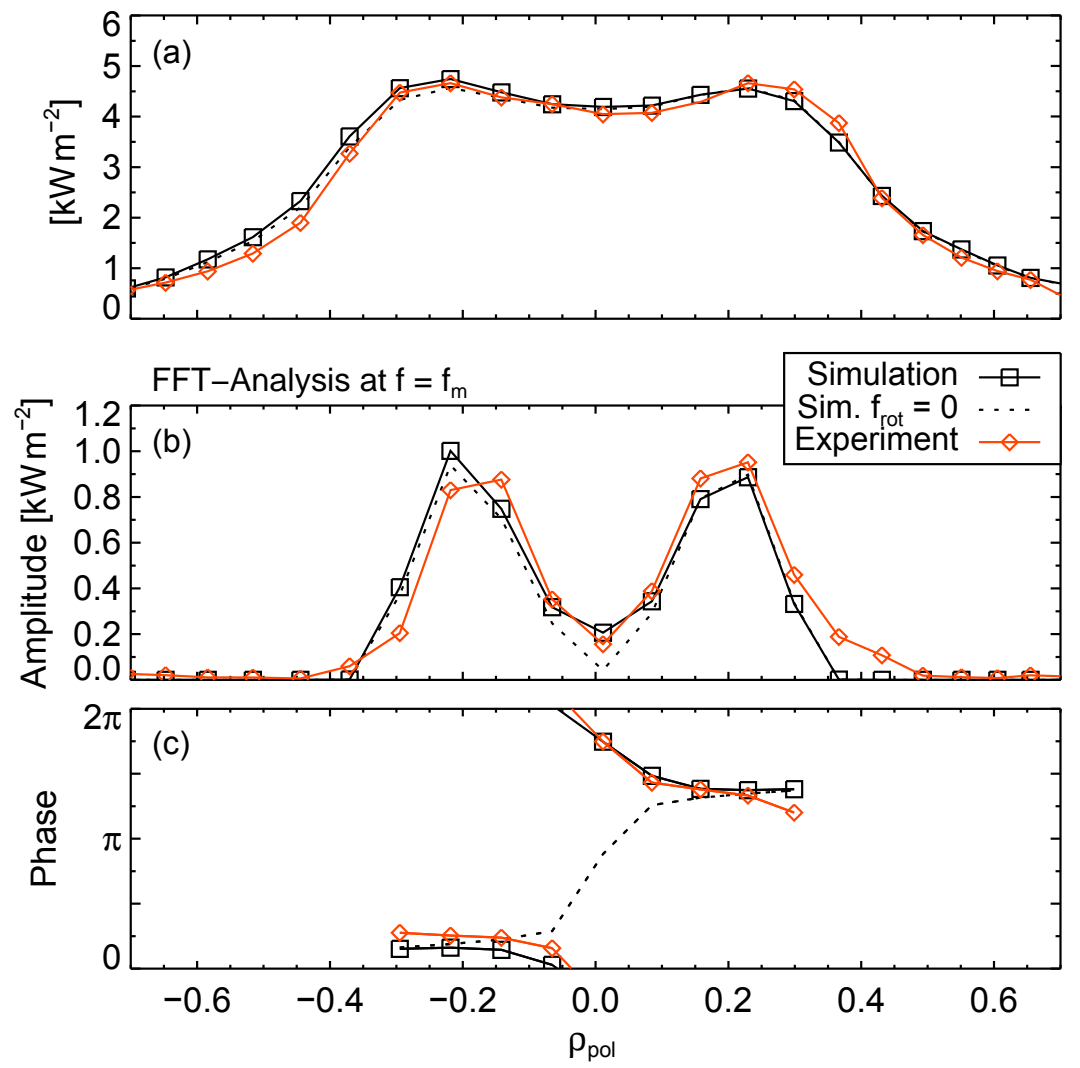

Figure 14: The comparison between experimental and forward-modeled profiles of a horizontal view (camera I) shows good agreement. (a): Time-averaged signals. (b)+(c): Amplitude and phase profile from a FFT analysis. In contrast, a forward-model neglecting the asymmetry in the SXR radiation (i.e. $f_{\text {rot }}=0 \mathrm{kHz}$ ) cannot reproduce the measured phase profile.

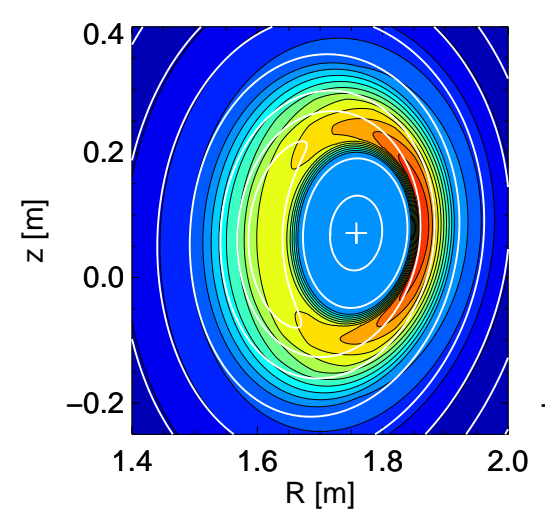

(a) $\Phi=0^{\circ}$

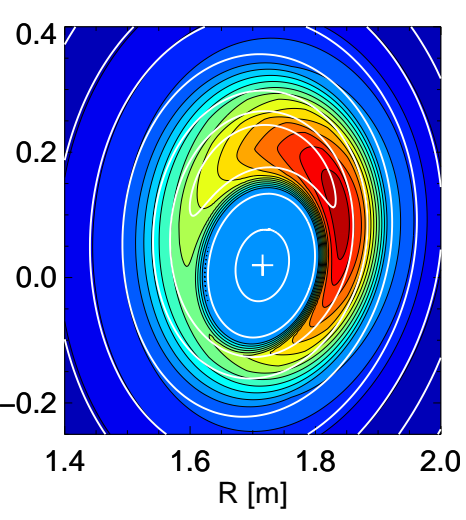

(b) $\Phi=90^{\circ}$

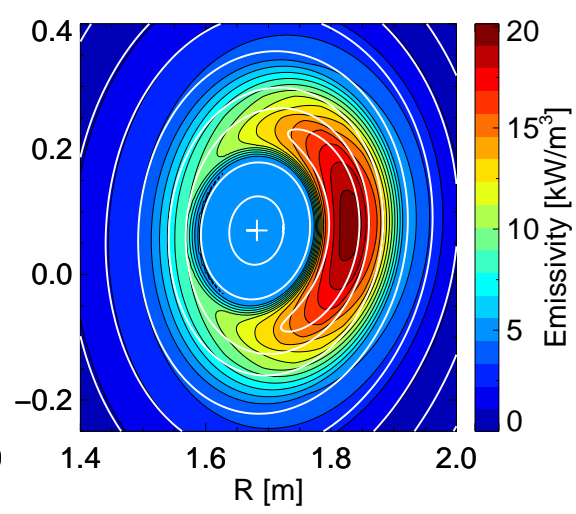

(c) $\Phi=180^{\circ}$

Figure 15: Contour plot of the modeled emissivity distribution (corresponding to the input profiles in figure 13 in three different mode phases $\Phi$. The helical mode flux surfaces are drawn with white lines, the cross indicates the displaced plasma center. 
flux-surface symmetric emissivity. In that case, the phase profile evolves in the opposite direction around the phase jump. Hence, we can conclude, that the shape of the phase profile can only be obtained correctly by including the asymmetry effects induced by rotation. Furthermore, the raising of the central minimum in the amplitude profile is also seen in the experimental data. As explained in the previous section, this is a confirmation that tungsten is also redistributed toroidally inside the mode structure.

The modeled emissivity resulting from the fit is shown in fig. 15 in the same three phases as the tomography (fig. 12). It can be seen, that the model-emissivity agrees with the tomography in the phases $\Phi=90^{\circ}$ and $\Phi=180^{\circ}$. In phase $\Phi=0^{\circ}$, there is a deviation in the narrow region around the island X-point, which may be explained by the finite spatial resolution of the tomography.

The simulation results for a vertical and an intermediate view are shown in figure 16 . Again, we find a very good agreement with the experimental data, although the input parameters were only fitted to the horizontal camera I. As predicted, the effect on the phase profile is weaker than for the horizontal view. The poloidal asymmetry between HFS and LFS, which can be seen in the time averaged profile and in the amplitude profile, is described very well. 

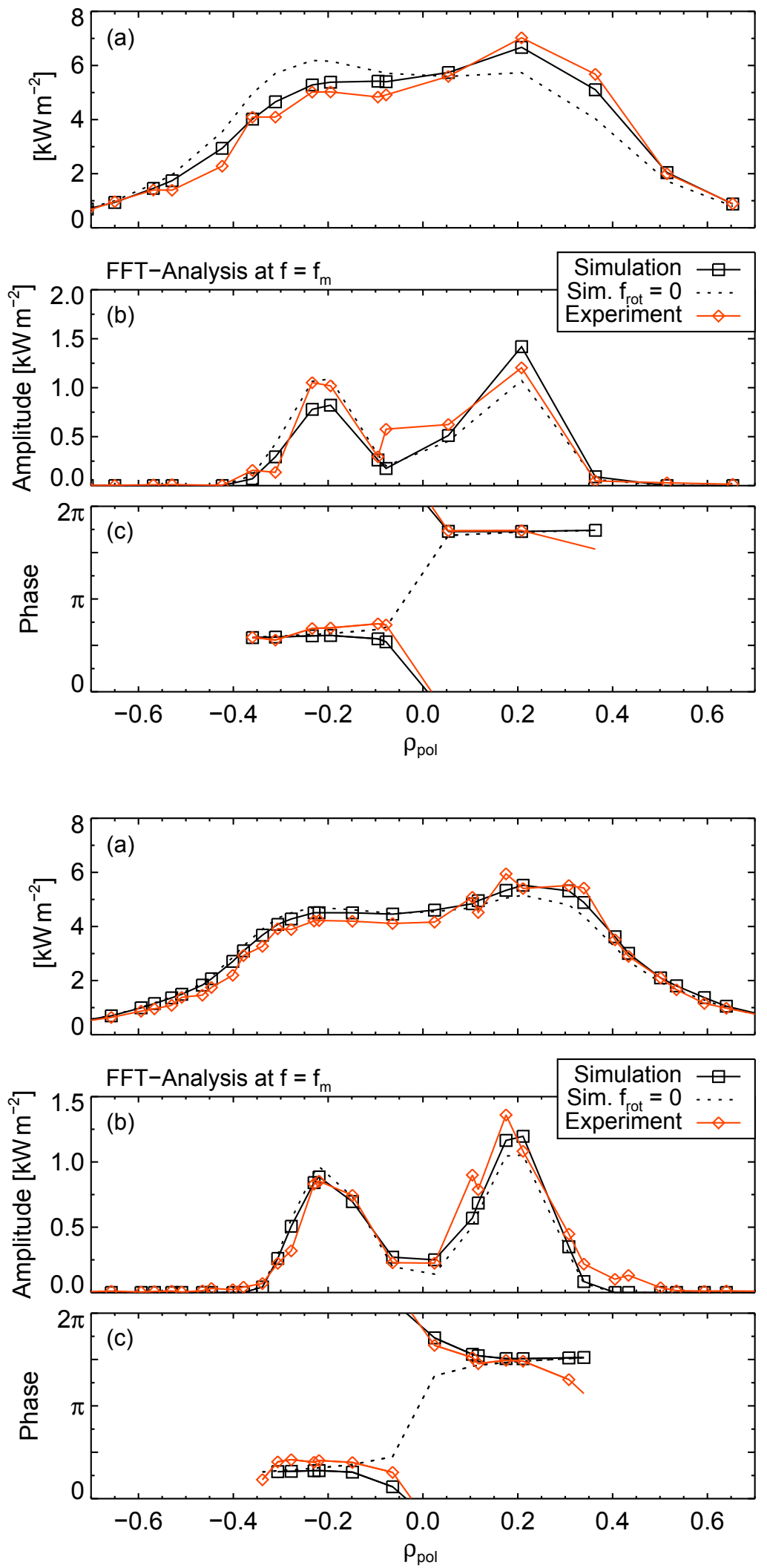

Figure 16: Comparison between experimental and forward-modeled profiles for a vertical view (camera $\mathrm{K}$ ) and a intermediate view (camera $\mathrm{J}$, down). (a): Time-averaged signals. (b) $+(\mathrm{c})$ : Amplitude and phase profile from a FFT analysis. 


\section{Summary}

A novel synthetic SXR diagnostic has been developed, which takes into account the full 3D geometry of the volumes of sight resulting from the detector-pinhole arrangement. A comparison with the more common approach of using lines of sight shows a significant difference in the simulated signals, which is due to the toroidal spreading of the VOS and the curvature of the plasma torus. The strength and sign of this difference also depends on the viewing direction. This must be taken into account in cases, where a precise calibration of the SXR diagnostics is needed - e.g. when doing a tomographic reconstruction.

With the synthetic diagnostic, the effect of three-dimensional SXR emissivity structures on the SXR signals is investigated. The 3D structure results from the tungsten density asymmetries in rotating plasmas together with the helical structure of MHD modes. Here, we have focused on the resistive $(1,1)$ kink, which is modeled with a helical flux model similar to [12]. This results in helical mode flux surfaces, for which we define a new radial coordinate $\rho_{\text {mod }}$. The SXR emissivity is then modeled in two parts: One part is constant on these surfaces, and resembles the SXR radiation from bremsstrahlung and light-Z impurity contributions. The second part is the SXR emissivity due to tungsten. In rotating plasmas, its density has a LFS-HFS asymmetry due to centrifugal forces. The $\mathrm{W}$ density distribution is then described by a force balance parallel to the flux surface [17, 18. We have assumed, that this force balance is still given inside the helically wound mode flux surfaces, which means that $\mathrm{W}$ can redistribute both poloidally and toroidally in the same manner. In order that such an equilibrium can build up, we had to assume that $\mathrm{W}$ rotates with the same velocity as the mode structure.

A measure for the strength of the SXR asymmetry is the toroidal rotation. By doing a rotation scan in our forward model, we could estimate the effect of asymmetries on the SXR signals in the presence of a $(1,1)$ mode and their FFT amplitude and phase profiles. The strongest effect is seen in the phase profile of horizontal views, which changes its slope sign around the phase jump with increasing strength of the asymmetry. Furthermore, a rise of the central amplitude minimum is observed in horizontal views due to the assumed toroidal redistribution.

Finally, our model is compared to experimental data from ASDEX Upgrade. We find a good agreement between forward modeling and the measured SXR signals, and the resulting FFT profiles. In particular, the shape of the phase profile is reproduced very well by our model, while it has the opposite shape when neglecting the tungsten asymmetry. This gives a good indication, that the tungsten distribution in helical mode structures can be well described by the force balance calculated in [18] for axisymmetric flux surfaces.

\section{Acknowledgment}

This work has been carried out within the framework of the EUROfusion Consortium and has received funding from the Euratom research and training programme 2014-2018 under grant agreement No 633053. The views and opinions expressed herein do not necessarily reflect those of the European Commission. 


\section{References}

[1] V. Igochine, S. Günter, M. Maraschek, and the ASDEX Upgrade Team. Investigation of complex MHD activity by a combined use of various diagnostics. Nuclear Fusion, 43(12):1801, 2003.

[2] J.S. Kim, L. Zhao, I.N. Bogatu, Y. In, A. Turnbull, T. Osborne, M. Maraschek, and K. Comer. Soft X-ray virtual diagnostics for tokamak simulations. Review of Scientific Instruments, 80(11):113503-113503, 2009.

[3] C. Angioni and P. Helander. Neoclassical transport of heavy impurities with poloidally asymmetric density distribution in tokamaks. Plasma Physics and Controlled Fusion, 56(12):124001, 2014.

[4] T. Pütterich, R. Dux, R. Neu, M. Bernert, M. N. A. Beurskens, V. Bobkov, S. Brezinsek, C. Challis, J. W. Coenen, I. Coffey, A. Czarnecka, C. Giroud, P. Jacquet, E. Joffrin, A. Kallenbach, M. Lehnen, E. Lerche, E. de la Luna, S. Marsen, G. Matthews, M.-L. Mayoral, R. M. McDermott, A. Meigs, J. Mlynar, M. Sertoli, G. van Rooij, the ASDEX Upgrade Team, and JET EFDA Contributors. Observations on the Wtransport in the core plasma of JET and ASDEX Upgrade. Plasma Physics and Controlled Fusion, 55(12):124036, 2013.

[5] F. J. Casson, C. Angioni, E. A. Belli, R. Bilato, P. Mantica, T. Odstrcil, T. Pütterich, M. Valisa, L. Garzotti, C. Giroud, J. Hobirk, C. F. Maggi, J. Mlynar, and M. L. Reinke. Theoretical description of heavy impurity transport and its application to the modelling of tungsten in JET and ASDEX Upgrade. Plasma Physics and Controlled Fusion, 57(1):014031, 2015.

[6] W. J. Karzas and R. Latter. Electron Radiative Transitions in a Coulomb Field. The Astrophysical Journal, Supplement, 6:167, May 1961.

[7] T. Pütterich, R. Neu, R. Dux, A.D. Whiteford, M.G. O’Mullane, H.P. Summers, and the ASDEX Upgrade Team. Calculation and experimental test of the cooling factor of tungsten. Nuclear Fusion, 50(2):025012 (9pp), 2010.

[8] M. Sertoli, V. Igochine, A. Gude, R. McDermott, and ASDEX Upgrade Team. Central impurity convection and its connection to MHD activity in ASDEX Upgrade. In A. Becoulet, T. Hoang, and U. Stroth, editors, Europhysics Conference Abstracts (CD-ROM, Proc. of the 38th EPS Conference on Plasma Physics, Strasbourg, France, 2011), volume $35 \mathrm{G}$ of ECA, page P5.116, Geneva, 2011. European Physical Society.

[9] V. Igochine, A. Gude, M. Maraschek, and ASDEX Upgrade Team. Hotlink based Soft X-ray Diagnostic on ASDEX Upgrade. Technical Report IPP 1/338, Max-PlanckInstitut für Plasmaphysik, Garching(DE), jan 2010.

[10] A. Van Oosterom and J. Strackee. The solid angle of a plane triangle. Biomedical Engineering, IEEE Transactions on, (2):125-126, 1983. 
[11] R. Bohle. Development of a virtual soft X-ray diagnostics and its application to ideal kink instabilities. Diploma thesis, LMU München, 2011.

[12] F. Porcelli, E. Rossi, G. Cima, and A. Wootton. Macroscopic magnetic islands and plasma energy transport. Phys. Rev. Lett., 82:1458-1461, Feb 1999.

[13] B. B. Kadomtsev. On disruptive instability in tokamaks. Soviet Journal of Plasma Physics, 1:710-715, October 1975.

[14] J. W. Connor and R. J. Hastie. The effect of shaped plasma cross sections on the ideal internal kink mode in a tokamak. Technical Report CLM-M106, Culham Laboratory, 1985.

[15] M. Schittenhelm and H. Zohm. Analysis of coupled MHD modes with Mirnov probes in ASDEX Upgrade. Nuclear Fusion, 37(9):1255, 1997.

[16] P.J. McCarthy, P. Martin, W. Schneider. The CLISTE interpretive equilibrium code. IPP Report 5/85, Max-Planck-Institut für Plasmaphysik, Garching, Germany, 1999.

[17] F. L. Hinton and S. K. Wong. Neoclassical ion transport in rotating axisymmetric plasmas. Physics of Fluids (1958-1988), 28(10):3082-3098, 1985.

[18] J.A. Wesson. Poloidal distribution of impurities in a rotating tokamak plasma. $\mathrm{Nu}$ clear Fusion, 37(5):577, 1997.

[19] M. Sokoll. MHD-Instabilitäten in magnetisch eingeschlossenen Plasmen und ihre tomographische Rekonstruktion im Röntgenlicht. Technical Report 1/309, IPP, Garching, Germany, April 1997.

[20] A. Gude. Identification of MHD Instabilities in Experiments. In V. Igochine, editor, Active Control of Magneto-hydrodynamic Instabilities in Hot Plasmas. Springer, Berlin Heidelberg, 2015.

[21] M. Odstrcil, J. Mlynar, T. Odstrcil, B. Alper, and A. Murari. Modern numerical methods for plasma tomography optimisation. Nuclear Instruments and Methods in Physics Research Section A: Accelerators, Spectrometers, Detectors and Associated Equipment, 686(0):156 - 161, 2012.

[22] A. Gude, M. Maraschek, C. Angioni, J. Stober, and ASDEX Upgrade Team. Hollow central radiation profiles and inverse sawtooth-like crashes in asdex upgrade plasmas with central wave heating. In C. McKenna, editor, Europhysics Conference Abstracts (CD-ROM, Proc. of the 37th EPS Conference on Plasma Physics, Dublin, Ireland, 2010), volume 34A of ECA, page P4.124, Geneva, 2010. European Physical Society. 


\section{A. Derivation of an analytical estimate for $R_{\mathrm{c}}$}

An analytical estimate of the shift of the poloidal projection of the VOS with respect to the LOS $R_{\text {los }}$ at a given $z$-position can be calculated by evaluating the center of mass $R_{\mathrm{c}}$ :

$$
R_{\mathrm{c}}(z)=\frac{\int R(u, v) \Omega(u, v, z) \mathrm{d} u \mathrm{~d} v}{\int \Omega(u, v, z) \mathrm{d} u \mathrm{~d} v} \approx \frac{\int R\left(u, R_{\mathrm{los}}\right) \Omega\left(u, R_{\mathrm{los}}, z\right) \mathrm{d} u}{\int \Omega\left(u, R_{\mathrm{los}}, z\right) \mathrm{d} u}
$$

$u$ and $v$ are coordinates along the toroidal and poloidal direction of the VOS in the $z$-plane (as shown in fig. 4(a)). In the second step, we have neglected the poloidal spreading of the VOS and assumed, that the center of the VOS goes through the torus axis (i.e. it lies in the poloidal plane). If the pinhole is centered over the diode in toroidal direction, $\Omega(u)$ has the shape of a symmetric trapezoid. Therefore, we parametrize it as (see also fig. $17(\mathrm{a})$ for the definition of the quantities):

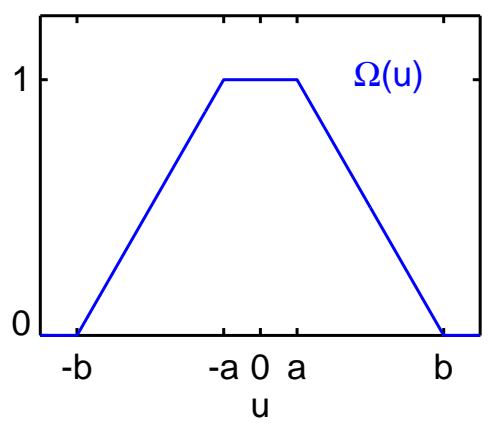

(a)

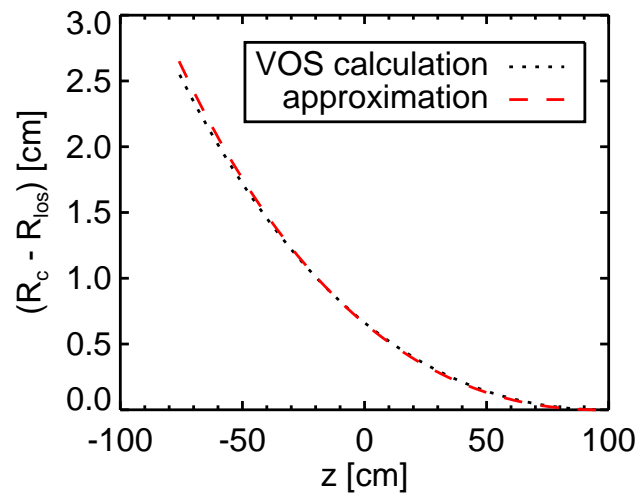

(b)

Figure 17: (a) The instrumental profile $\Omega$ is parametrized in the toroidal direction $u$ as a symmetrical trapezoid with the parameters $a$ and $b$. The full toroidal width of the VOS is $\Delta_{\mathrm{t}}=2 b$, and we write the ratio between $a$ and $b$ as $\delta=\frac{a}{b}$. (b) The radial shift $R_{\mathrm{c}}-R_{\text {los }}$ as a function of $z$ along the line of sight H_050. The black dotted line refers to the numerical VOS calculation, and the dashed red line corresponds to the approximate formula 13 .

$$
\Omega(u)= \begin{cases}1 & |u| \in[0 ; a] \\ \frac{|u|-b}{a-b}=\frac{|u|-b}{(\delta-1) b} & |u| \in[a ; b] \\ 0 & \text { else }\end{cases}
$$

Then, the integral in the denominator gives:

$$
\int \Omega(u) \mathrm{d} u=b(1+\delta)
$$


For the integral in the nominator, we do a Taylor-approximation of the square root:

$$
\int \Omega(u) R \mathrm{~d} u=\int \Omega(u) \sqrt{R_{\operatorname{los}}^{2}+u^{2}} \mathrm{~d} u \approx \int \Omega(u) R_{\operatorname{los}}\left(1+\frac{u^{2}}{2 R_{\operatorname{los}}^{2}}\right) \mathrm{d} u
$$

Then, the integral can be solved straight-forward and we get:

$$
R_{\mathrm{C}}=R_{\mathrm{los}}+\frac{\Delta_{\mathrm{t}}^{2}}{48 R_{\mathrm{los}}}\left(1+\delta^{2}\right)
$$

Herein, $\Delta_{\mathrm{t}}=2 b$ is the full toroidal width of the VOS in the $z$-plane, and $\delta=a / b$ with $0 \leq \delta \leq 1 . \delta=0$ corresponds to a triangular $\Omega(u)$, and $\delta=1$ corresponds to the case, that $\Omega(u)$ is a box function.

Now, we can calculate $\delta$ and $\Delta_{\mathrm{t}}$ for a point on the LOS with distance $l$ from the pinhole by geometric considerations. In the limit, that $l \gg d$ with $d$ being the distance between diode and pinhole, $\delta$ can be calculated from the toroidal pinhole and diode width $\left(P_{\mathrm{t}}\right.$ and $\left.D_{\mathrm{t}}\right)$ :

$$
\delta=\frac{\arctan \frac{\left|D_{\mathrm{t}}-P_{\mathrm{t}}\right|}{2 d}}{\arctan \frac{D_{\mathrm{t}}+P_{\mathrm{t}}}{2 d}}
$$

Hereby, $\gamma=2 \arctan \frac{D_{\mathrm{t}}+P_{\mathrm{t}}}{2 d}$ is the full opening angle of the VOS. $\Delta_{\mathrm{t}}$ can be calculated by:

$$
\Delta_{\mathrm{t}}=2 \tan \frac{\gamma}{2} \cdot\left(l+d \frac{P_{\mathrm{t}}}{D_{\mathrm{t}}+P_{\mathrm{t}}}\right) \cdot \cos \alpha_{\mathrm{p}}
$$

where $\alpha_{\mathrm{p}}$ is the poloidal angle between the diode surface normal and the LOS.

For the ASDEX Upgrade SXR cameras $\left(D_{\mathrm{t}}=4.6 \mathrm{~mm}, P_{\mathrm{t}}=5.0 \mathrm{~mm}, d=14.0 \mathrm{~mm}\right)$ we find $\delta=0.043$, i.e. $\Omega(u)$ is almost triangular. In fig. $17(\mathrm{~b})$, the radial shift $R_{\mathrm{c}}-R_{\text {los }}$ is shown for signal H_050 (see fig 4(b) as a function of $z$, calculated with equation 13 . The comparison with the numerical VOS simulation shows a good agreement, which can also be seen as a proof, that the deviations between LOS and VOS calculations discussed in section 3 are indeed dominantly caused by the toroidal spreading of the VOS. 